\title{
POSITIVE SOLUTIONS FOR THE PERIODIC SCALAR $p$-LAPLACIAN: EXISTENCE AND UNIQUENESS
}

\author{
Sophia Th. Kyritsi and Nikolaos S. Papageorgiou
}

\begin{abstract}
We study a nonlinear periodic problem driven by the scalar $p$-Laplacian. The reaction term is a Caratheodory function $f(t, x)$ which satisfies only a unilateral growth condition in the $x$-variable. Assuming strict monotonicity for the quotient $f(t, x) / x^{p-1}$ and using variational methods coupled with suitable truncation techniques, we produce necessary and sufficient conditions for the existence and uniqueness of positive solutions.
\end{abstract}

\section{INTRODUCTION}

In this paper we study the following nonlinear periodic problem driven by the scalar $p$-Laplacian:

$$
\left\{\begin{array}{c}
-\left(\left|u^{\prime}(t)\right|^{p-2} u^{\prime}(t)\right)^{\prime}=f(t, u(t)) \quad \text { a.e. on } T=[0, b], \\
u(0)=u(b), u^{\prime}(0)=u^{\prime}(b), u \geq 0,1<p<\infty
\end{array}\right\} .
$$

The reaction term $f(t, x)$ is a Carathéodory function, i.e., for all $x \in \mathbb{R}, t \longrightarrow f(t, x)$ is measurable, and for a.a. $t \in T, x \longrightarrow f(t, x)$ is continuous.

The aim of this work is to establish the existence and uniqueness of positive solutions, when the nonlinearity $f(t, \cdot)$ is only unilaterally restricted (only from above). In fact, we produce necessary and sufficient conditions for such problems to have a unique positive solution.

There have been papers dealing with the existence and multiplicity of positive solutions for the periodic scalar $p$-Laplacian. We mention the works of AizicoviciPapageorgiou-Staicu [1], Binding-Rynne [3, 4], del Pino-Manásevich-Murúa [5], Drabek-Manásevich [6], Kyritsi-Papageorgiou [7], Motreanu-Motreanu-Papageorgiou [8], Yang [11], and Zhang [12]. In all these works a bilateral polynomial growth is

Received April 4, 2011, accepted August 29, 2011.

Communicated by Biagio Ricceri.

2010 Mathematics Subject Classification: 34B15, 34B18, 34C25.

Key words and phrases: Scalar $p$-Laplacian, Unilateral growth, Existence and uniqueness of positive solutions, Weighted eigenvalues. 
imposed on the right-hand side. To the best of our knowledge, the question of existence and uniqueness of periodic solutions for the scalar $p$-Laplacian has not been addressed in this generality.

Our approach is variational with suitable truncation techniques.

\section{Mathematical Background}

We start by considering the following weighted nonlinear eigenvalue problem:

$$
\left\{\begin{array}{c}
-\left(\left|u^{\prime}(t)\right|^{p-2} u^{\prime}(t)\right)^{\prime}=(\widehat{\lambda}+\beta(t))|u(t)|^{p-2} u(t) \quad \text { a.e. on } T=[0, b], \\
u(0)=u(b), u^{\prime}(0)=u^{\prime}(b), 1<p<\infty, \widehat{\lambda} \in \mathbb{R}, \beta \in L^{1}(T)
\end{array}\right\} .
$$

This eigenvalue problem was first investigated by Zhang [12] and later BindingRynne [3, 4] answered important questions left open by Zhang and produced a more definitive picture for the spectrum of problem (2). In particular, from Binding-Rynne [4], we know that problem (2) admits a smallest eigenvalue $\widehat{\lambda}_{0}(\beta) \in \mathbb{R}$ which is simple and has the following variational characterization:

$$
\widehat{\lambda}_{0}(\beta)=\inf \left[\left\|u^{\prime}\right\|_{p}^{p}-\int_{0}^{b} \beta(t)|u(t)|^{p} \mathrm{~d} t: u \in W_{\text {per }}^{1, p}(0, b),\|u\|_{p}=1\right]
$$

where $W_{\text {per }}^{1, p}(0, b)=\left\{u \in W^{1, p}(0, b): u(0)=u(b)\right\}$. Recall that $W^{1, p}(0, b)$ is embedded continuously, (in fact compactly), into $C(T)$, therefore the evaluations at $t=0$ and $t=b$ in the definition of $W_{\text {per }}^{1, p}(0, b)$ make sense. Every eigenfunction $u \in W_{\text {per }}^{1, p}(0, b)$ corresponding to $\widehat{\lambda}_{0}(\beta)$ satisfies

$$
u \in C_{0}^{1}(Z) 1(T) \text { and }|u(t)|>0 \text { for all } t \in T .
$$

So, an eigenfunction corresponding to $\widehat{\lambda}_{0}(\beta)$ has constant sign and we can always assume that it is positive. An eigenfunction corresponding to an eigenvalue $\widehat{\lambda} \neq \widehat{\lambda}_{1}(\beta)$ is necessarily nodal (i.e., sign changing).

We can rewrite (3) as follows:

$$
\widehat{\lambda}_{0}(\beta)=\inf \left[\left\|u^{\prime}\right\|_{p}^{p}-\int_{\{u \neq 0\}} \beta(t)|u(t)|^{p} \mathrm{~d} t: u \in W_{\text {per }}^{1, p}(0, b),\|u\|_{p}=1\right] .
$$

We observe that in (4), the integral $\int_{\{u \neq 0\}} \beta|u|^{p} \mathrm{~d} t$ makes sense even when $\beta(\cdot)$ is only a measurable function and there exists $\widehat{c} \in L^{1}(T)_{+}$such that

$$
\beta(t) \leq \widehat{c}(t) \text { a.e. on } T \text { or } \beta(t) \geq-\widehat{c}(t) \text { a.e. on } T \text {. }
$$

In the first case $\widehat{\lambda}_{0}(\beta) \in(-\infty,+\infty]$ and in the second case $\widehat{\lambda}_{0}(\beta) \in[-\infty,+\infty)$. 
In addition to the Sobolev space $W_{\text {per }}^{1, p}(0, b)$, we shall also use the Banach space $\widehat{C}^{1}(T)=C^{1}(T) \cap W_{\text {per }}^{1, p}(0, b)=\left\{u \in C^{1}(T): u(0)=u(b)\right\}$. This is an ordered Banach space with an order cone given by

$$
\widehat{C}_{+}=\left\{u \in \widehat{C}^{1}(T): u(t) \geq 0 \text { for all } t \in T\right\} .
$$

This cone has an nonempty interior given by

$$
\operatorname{int} \widehat{C}_{+}=\left\{u \in \widehat{C}_{+}: u(t)>0 \text { for all } t \in T\right\} .
$$

Let $u, v \in \operatorname{int} \widehat{C}_{+}$and set

$$
R(u, v)=\left|u^{\prime}(t)\right|^{p}-\left|v^{\prime}(t)\right|^{p-2} v^{\prime}(t)\left(\frac{u(t)^{p}}{v(t)^{p-1}}\right)^{\prime} .
$$

From Allegretto-Huang [2] we know that $R(u, v)(t) \geq 0$ for all $t \in T$.

Let $A: W_{\text {per }}^{1, p}(0, b) \longrightarrow W_{\text {per }}^{1, p}(0, b)^{*}$ be the nonlinear operator defined by

$$
\langle A(u), y\rangle=\int_{0}^{b}\left|u^{\prime}\right|^{p-2} u^{\prime} y^{\prime} \mathrm{d} t \quad \text { for all } u, y \in W_{\text {per }}^{1, p}(0, b)
$$

(by $\langle\cdot, \cdot\rangle$ we denote the duality brackets for the pair $\left.\left(W_{\text {per }}^{1, p}(0, b)^{*}, W_{\text {per }}^{1, p}(0, b)\right)\right)$. This map is continuous and monotone (see, for example, Papageorgiou-Kyritsi [9]).

Throughout this work by $\|\cdot\|$ we denote the norm of the Sobolev space $W_{\text {per }}^{1, p}(0, b)$ and for $p \in[1, \infty]$, by $\|\cdot\|_{p}$ we denote the norm of the Lebesgue space $L^{p}(T)$. Finally, for every $r \in \mathbb{R}$, we set $r^{ \pm}=\max \{ \pm r, 0\}$ and by $|\cdot|_{1}$ we denote the Lebesgue measure on $\mathbb{R}$.

\section{Existence of Positive Solutions}

The hypotheses on the reaction term $f(t, x)$ are the following:

H: $f: T \times \mathbb{R} \longrightarrow \mathbb{R}$ is a Carathéodory function such that

(i) for all $x \geq 0, f(\cdot, x) \in L^{1}(T)$ and there exists $\alpha \in L^{1}(T)_{+}$such that

$$
f(t, x) \leq \alpha(t)\left(1+x^{p-1}\right) \text { for a.a } t \in T, \text { all } x \geq 0 ;
$$

(ii) for a.a. $t \in T$, the function $x \longrightarrow \frac{f(t, x)}{x^{p-1}}$ is strictly decreasing on $(0,+\infty)$;

(iii) if $\vartheta(t)=\lim _{x \rightarrow+\infty} \frac{f(t, x)}{x^{p-1}}$, then $\widehat{\lambda}_{0}(\vartheta)>0$;

(iv) if $\vartheta_{0}(t)=\lim _{x \rightarrow 0^{+}} \frac{f(t, x)}{x^{p-1}}$, then $\widehat{\lambda}_{0}\left(\vartheta_{0}\right)<0$; 
Remark 3.1. Because we are looking for positive solutions and the hypotheses on $f(t, \cdot)$ concern only the positive semiaxis $\mathbb{R}_{+}=[0,+\infty)$, by truncating $f(t, \cdot)$ if necessary, we may (and will) assume that $f(t, x)=f(t, 0)$ for a.a. $t \in T$, all $x \leq 0$. By virtue of hypothesis $\mathrm{H}(\mathrm{ii})$ we see that the limits $\vartheta(t), \vartheta_{0}(t)$ in hypotheses $\mathrm{H}(\mathrm{iii})$, (iv) exist and are measurable functions. We have

$$
\begin{aligned}
& \frac{f(t, x)}{x^{p-1}} \leq f(t, 1) \text { for a.a. } t \in T, \text { all } x \geq 1 \text { and } f(\cdot, 1) \in L^{1}(T), \\
\Rightarrow & \vartheta(t) \leq f(t, 1) \text { for a.a. } t \in T, \\
\Rightarrow & \widehat{\lambda}_{0}(\vartheta) \in(-\infty,+\infty] .
\end{aligned}
$$

Similarly, we have

$$
\begin{aligned}
& \frac{f(t, x)}{x^{p-1} \geq f(t, 1) \text { for a.a. } t \in T, \text { all } x \in(0,1] \text { and } f(\cdot, 1) \in L^{1}(T),} \\
\Rightarrow & \vartheta_{0}(t) \geq f(t, 1) \text { for a.a. } t \in T, \\
\Rightarrow & \widehat{\lambda}_{0}\left(\vartheta_{0}\right) \in[-\infty,+\infty) .
\end{aligned}
$$

If $\vartheta, \vartheta_{0} \in L^{1}(T)$, then $\widehat{\lambda}_{0}(\vartheta), \widehat{\lambda}_{0}\left(\vartheta_{0}\right) \in \mathbb{R}$ and are the principal eigenvalues of (2) when $\beta=\vartheta$ and $\beta=\vartheta_{0}$ respectively. In the autonomous case, i.e., when $f(t, x)=f \varnothing$, hypotheses $\mathrm{H}$ (iii), (iv) reduce to

$$
\vartheta_{0}<0<\vartheta
$$

(recall that 0 is the principal eigenvalue of the negative periodic scalar $p$-Laplacian, i.e., for problem (2) when $\beta \equiv 0$ ).

Example 3.2. The function $f \varnothing=\lambda\left(x^{r-1}-x^{q-1}\right)$ for all $x \geq 0$ with $\lambda>0$, $1<r \leq p \leq q<\infty$, and $r \neq p$ or $q \neq p$, satisfies hypotheses $\mathrm{H}$. In this case $\vartheta=-\infty$ if $q>p$ and $\vartheta=-\lambda$ if $r<p=q$ (and thus $\widehat{\lambda}_{0}(\vartheta)=+\infty$ if $q>p$ and $\widehat{\lambda}_{0}(\vartheta)=\lambda$ if $r<p=q$ ) and $\vartheta_{0}=+\infty$ if $r<p$ and $\vartheta_{0}=\lambda$ if $r=p<q$ (and thus $\hat{\lambda}_{0}\left(\vartheta_{0}\right)=-\infty$ if $r<p$ and $\widehat{\lambda}\left(\vartheta_{0}\right)=-\lambda$ if $\left.r=p<q\right)$. Another admissible nonlinearity is provided by the $f \varnothing=x^{r-1}-\delta x^{p-1} e^{x}$ for all $x \geq 0, \delta>0$, which does not exhibit a polynomial growth from below.

We introduce the following truncation-perturbation of $f(t, x)$ :

$$
\widehat{f}(t, x)=\left\{\begin{array}{ll}
f(t, 0), & \text { if } x \leq 0 \\
f(t, x)+x^{p-1} & \text { if } x>0
\end{array} .\right.
$$

This is a Caratheodory function. Let $\widehat{F}(t, x)=\int_{0}^{b} \widehat{f}(t, s) \mathrm{d} s$. Hypothesis H(i) and (5) imply that

$$
\widehat{F}(t, x) \leq \alpha_{1}(t)\left(1+x^{p}\right) \text { for a.a. } t \in T, \text { all } x \geq 0 \text { with } \alpha_{1} \in L^{1}(T)_{+} .
$$


This growth restriction on $\widehat{F}(t, \cdot)$, the fact that $f(\cdot, x) \in L^{1}(T)$ for all $x \geq 0$, and hypothesis $\mathrm{H}(\mathrm{ii})$ permit the introduction of the functional $\widehat{\varphi}: W_{\text {per }}^{1, p}(0, b) \longrightarrow \mathbb{R}$ defined by

$$
\widehat{\varphi}(u)=\frac{1}{p}\left\|u^{\prime}\right\|_{p}^{p}+\frac{1}{p}\|u\|_{p}^{p}-\int_{0}^{b} \widehat{F}(t, u(t)) \mathrm{d} t \quad \text { for all } u \in W_{\text {per }}^{1, p}(0, b) .
$$

Proposition 3.3. If hypotheses $H$ hold, then $\widehat{\varphi}$ is coercive, i.e., $\widehat{\varphi}(u) \longrightarrow+\infty$ as $\|u\| \rightarrow \infty$.

Proof. We argue indirectly. So, suppose that the result is not true. Then we can find $\left\{u_{n}\right\}_{n \geq 1} \subseteq W_{\text {per }}^{1, p}(0, b)$ such that

$$
\left\|u_{n}\right\| \longrightarrow \infty \text { and } \widehat{\varphi}\left(u_{n}\right) \leq M_{1} \text { for some } M_{1}>0, \text { all } n \geq 1 .
$$

We have

$$
\frac{1}{p}\left(\left\|u_{n}^{\prime}\right\|_{p}^{p}+\left\|u_{n}\right\|_{p}^{p}\right) \leq M_{1}+\int_{0}^{b} \widehat{F}\left(t, u_{n}\right) \mathrm{d} t \quad \text { for all } n \geq 1
$$

Note that

$$
\widehat{F}\left(t, u_{n}(t)\right)=\widehat{F}\left(t, u_{n}^{+}(t)\right)+f(t, 0)\left(-u_{n}^{-}(t)\right) \text { for a.a. } t \in T, \text { all } n \geq 1
$$

$($ see $(5))$

and $\frac{f(t, x)}{x^{p-1}} \geq f(t, 1)$ for a.a. $t \in T$, all $x \in(0,1]$,

$$
\Rightarrow f(t, x) \geq f(t, 1) x^{p-1} \text { for a.a. } t \in T, \text { all } x \in(0,1],
$$

(9) $\Rightarrow f(t, 0) \geq 0$ for a.a. $t \in T$.

It follows that

$$
\widehat{F}\left(t, u_{n}(t)\right) \leq \widehat{F}\left(t, u_{n}^{+}(t)\right) \leq \alpha(t)\left(1+\left|u_{n}(t)\right|^{p}\right) \text { a.a. } t \in T,
$$

(see [6]).

So, if we use this fact in (8), then

$$
\begin{aligned}
\frac{1}{p}\left(\left\|u_{n}^{\prime}\right\|_{p}^{p}+\left\|u_{n}\right\|_{p}^{p}\right) & \leq M_{1}+\int_{0}^{b} \widehat{F}\left(t, u_{n}^{+}\right) \mathrm{d} t \\
& \leq c_{1}\left(1+\left\|u_{n}\right\|_{\infty}^{p}\right) \text { for some } c_{1}>0, \text { all } n \geq 1 .
\end{aligned}
$$

From (10) and since $\left\|u_{n}\right\| \rightarrow \infty$ (see (7)), we see that $\left\|u_{n}\right\|_{\infty} \rightarrow \infty$. Let $y_{n}=$ $\frac{u_{n}}{\left\|u_{n}\right\|_{\infty}}, n \geq 1$. Then $\left\|y_{n}\right\|_{\infty}=1$ for all $n \geq 1$ and from (10), we have

$$
\begin{aligned}
& \frac{1}{p}\left(\left\|y_{n}^{\prime}\right\|_{p}^{p}+\left\|y_{n}\right\|_{p}^{p}\right) \leq c_{1}\left(\frac{1}{\left\|u_{n}\right\|_{\infty}^{p}}+1\right) \text { for all } n \geq 1, \\
\Rightarrow & \left\{y_{n}\right\}_{n \geq 1} \subseteq W_{\text {per }}^{1, p}(0, b) \text { is bounded. }
\end{aligned}
$$


By passing to a suitable subsequence if necessary, we may assume that

$$
y_{n} \stackrel{w}{\longrightarrow} y \text { in } W_{\text {per }}^{1, p}(0, b) \text { and } y_{n} \longrightarrow y \text { in } C(T) \text { with }\|y\|_{\infty}=1 .
$$

If $F(t, x)=\int_{0}^{x} f(t, s) \mathrm{d} s$, then from (7) we have

$$
\begin{aligned}
& \frac{1}{p}\left(\left\|y_{n}^{\prime}\right\|_{p}^{p}+\left\|y_{n}\right\|_{p}^{p}\right) \leq \frac{M_{1}}{\left\|u_{n}\right\|_{\infty}^{p}}+\int_{0}^{b} \frac{\widehat{F}\left(t, u_{n}\right)}{\left\|u_{n}\right\|_{\infty}^{p}} \mathrm{~d} t \\
= & \frac{M_{1}}{\left\|u_{n}\right\|_{\infty}^{p}}+\int_{\left\{u_{n}>0\right\}}\left(\frac{F\left(t, u_{n}\right)}{\left\|u_{n}\right\|_{\infty}^{p}}+\frac{1}{p} y_{n}^{p}\right) \mathrm{d} t \\
& +\int_{\left\{u_{n} \leq 0\right\}} \frac{f(t, 0)}{\left\|u_{n}\right\|_{\infty}^{p}} u_{n} \mathrm{~d} t \quad(\text { see }(5)) \\
\leq & \frac{M_{1}}{\left\|u_{n}\right\|_{\infty}^{p}}+\frac{1}{p}\left\|y_{n}^{+}\right\|_{\infty}^{p}+\int_{0}^{b} \frac{F\left(t, u_{n}^{+}\right)}{\left\|u_{n}\right\|_{\infty}^{p}} \mathrm{~d} t \quad \text { for all } n \geq 1
\end{aligned}
$$

(see (9)).

First assume that $\left\{u_{n}^{+}\right\}_{n \geq 1} \subseteq C(T)$ is bounded. Then $y \leq 0$. Hypothesis H(i) implies that

$$
F(t, x) \leq \alpha_{2}(t)\left(1+x^{p}\right) \text { for a.a. } t \in T, \text { all } x \geq 0 \text { with } \alpha_{2} \in L^{1}(T)_{+} .
$$

So, we have

$$
\begin{gathered}
\int_{0}^{b} \frac{F\left(t, u_{n}^{+}\right)}{\left\|u_{n}\right\|_{\infty}^{p}} \mathrm{~d} t \leq \int_{0}^{b} \alpha_{2}(t)\left(\frac{1}{\left\|u_{n}\right\|_{\infty}^{p}}+\left(y_{n}^{+}\right)^{p}\right) \mathrm{d} t \quad(\text { see }(13)) \\
\leq c_{2}\left(\frac{1}{\left\|u_{n}\right\|_{\infty}^{p}}+\left\|y_{n}^{+}\right\|_{\infty}^{p}\right) \text { for some } c_{2}>0, \text { all } n \geq 1, \\
\Rightarrow \limsup _{n \rightarrow \infty} \int_{0}^{b} \frac{F\left(t, u_{n}^{+}\right)}{\left\|u_{n}\right\|_{\infty}^{p}} \mathrm{~d} t \leq 0 \quad(\text { see }(11) \text { and recall } y \leq 0) .
\end{gathered}
$$

Then passing to the limit as $n \rightarrow \infty$ in (12) and using (11), we obtain

$$
\begin{aligned}
& \frac{1}{p}\left(\left\|y_{n}^{\prime}\right\|_{p}^{p}+\|y\|_{p}^{p}\right) \leq 0, \\
\Rightarrow & y=0, \text { which contradicts (11). }
\end{aligned}
$$

Hence we may assume that $\left\|u_{n}^{+}\right\| \rightarrow \infty$. From (5) we have

$$
\frac{1}{p}\left\|\left(y_{n}^{+}\right)^{\prime}\right\|_{p}^{p} \leq \frac{M_{1}}{\left\|u_{n}^{+}\right\|_{\infty}^{p}}+\int_{0}^{b} \frac{F\left(t, u_{n}^{+}\right)}{\left\|u_{n}^{+}\right\|_{\infty}^{p}} \mathrm{~d} t \quad \text { for all } n \geq 1 \quad \text { (see (9)). }
$$


Since $F(t, 0)=0$, we have

$$
\int_{0}^{b} \frac{F\left(t, u_{n}^{+}\right)}{\left\|u_{n}^{+}\right\|_{\infty}^{p}} \mathrm{~d} t=\int_{\left\{y^{+}=0\right\}} \frac{F\left(t, u_{n}^{+}\right)}{\left\|u_{n}^{+}\right\|_{\infty}^{p}} \mathrm{~d} t+\int_{\{y>0\} \cap\left\{y_{n}>0\right\}} \frac{F\left(t, u_{n}^{+}\right)}{\left(u_{n}^{+}\right)^{p}}\left(y_{n}^{+}\right)^{p} \mathrm{~d} t
$$

for all $n \geq 1$.

We know that $y_{n}^{+} \longrightarrow y^{+}$in $C(T)$ (see (11)). Therefore, using (13) we have

$$
\left|\int_{\left\{y^{+}=0\right\}} \frac{F\left(t, u_{n}^{+}\right)}{\left\|u_{n}^{+}\right\|_{\infty}^{p}} \mathrm{~d} t\right| \leq \int_{\left\{y^{+}=0\right\}} \alpha_{2}(t)\left(\frac{1}{\left\|u_{n}^{+}\right\|_{\infty}^{p}}+\left(y_{n}^{+}\right)^{p}\right) \mathrm{d} t \longrightarrow 0 \text { as } n \rightarrow \infty .
$$

Note that

$$
u_{n}^{+}(t) \longrightarrow+\infty \text { for all } t \in\{y>0\} \text { and } \chi_{\{y>0\} \cap\left\{y_{n}>0\right\}}(t) \longrightarrow \chi_{\{y>0\}}(t)
$$

for a.a. $t \in T$.

Here by $\chi_{A}$ we indicate the characteristic function of a set $A \subseteq T$, i.e.,

$$
\chi_{A}(t)=\left\{\begin{array}{ll}
1 & \text { if } t \in A \\
0 & \text { if } t \in T \backslash A
\end{array} .\right.
$$

Let $t \in\{\vartheta>-\infty\} \backslash N,|N|_{1}=0$ be such that $\frac{f(t, x)}{x^{p-1}} \longrightarrow \vartheta(t)^{+}$as $x \rightarrow+\infty$ (see hypotheses $\mathrm{H}(\mathrm{ii})$ and (iii)). Then given any $\varepsilon>0$ we can find $M_{2}=M_{2}(\varepsilon, t)>0$ such that

$$
\begin{aligned}
& f(t, x) \leq(\vartheta(t)+\varepsilon) x^{p-1} \quad \text { for all } x \geq M_{2}, \\
\Rightarrow & F(t, x) \leq \frac{1}{p}(\vartheta(t)+\varepsilon) x^{p} \quad \text { for all } x \geq M_{2}, \\
\Rightarrow & \frac{F(t, x)}{x^{p}} \leq \frac{1}{p}(\vartheta(t)+\varepsilon) \quad \text { for all } x \geq M_{2}, \\
\Rightarrow & \limsup _{x \rightarrow+\infty} \frac{F(t, x)}{x^{p}} \leq \frac{1}{p}(\vartheta(t)+\varepsilon) .
\end{aligned}
$$

Since $\varepsilon>0$ was arbitrary, we let $\varepsilon \longrightarrow 0^{+}$to infer that

$$
\limsup _{x \rightarrow+\infty} \frac{F(t, x)}{x^{p}} \leq \frac{1}{p} \vartheta(t) \text { for a.a. } t \in\{\vartheta>-\infty\}
$$

Also, if $t \in\{\vartheta=-\infty\} \backslash N,|N|_{1}=0$ is such that $\frac{f(t, x)}{x^{p-1}} \longrightarrow-\infty=\vartheta(t)$ as $x \rightarrow+\infty$, then for every $\xi>0$, we can find $M_{3}=M_{3}(\xi, t)>0$ such that

$$
\begin{aligned}
& f(t, x) \leq-\xi x^{p-1} \quad \text { for all } x \geq M_{3}, \\
\Rightarrow & \frac{F(t, x)}{x^{p}} \leq-\frac{\xi}{p} \text { for all } x \geq M_{3}, \\
\Rightarrow & \limsup _{x \rightarrow+\infty} \frac{F(t, x)}{x^{p}} \leq-\frac{\xi}{p} \text { for a.a. } t \in\{\vartheta=-\infty\} .
\end{aligned}
$$


Since $\xi$ was arbitrary, we let $\xi \rightarrow+\infty$ and have

$$
\lim _{x \rightarrow+\infty} \frac{F(t, x)}{x^{p}}=-\infty=\frac{\vartheta(t)}{p} \text { for a.a. } t \in\{\vartheta=-\infty\} .
$$

Therefore, finally we have

$$
\limsup _{x \rightarrow+\infty} \frac{F(t, x)}{x^{p}} \leq \frac{1}{p} \vartheta(t) \text { a.e. on } T .
$$

Because of (13) we can use Fatou's lemma and (17) and obtain

$$
\begin{aligned}
& \limsup _{n \rightarrow \infty} \int_{\{y>0\} \cap\left\{y_{n}>0\right\}} \frac{F\left(t, u_{n}^{+}\right)}{\left(u_{n}^{+}\right)^{p}}\left(y_{n}^{+}\right)^{p} \mathrm{~d} t \\
\leq & \frac{1}{p} \int_{\{y>0\}} \vartheta y^{p} \mathrm{~d} t=\frac{1}{p} \int_{\left\{y^{+} \neq 0\right\}} \vartheta\left(y^{+}\right)^{p} \mathrm{~d} t .
\end{aligned}
$$

So, if in (15) we pass to the limit as $n \rightarrow \infty$ and use (16) and (18), we have

$$
\limsup _{n \rightarrow \infty} \int_{0}^{b} \frac{F\left(t, u_{n}^{+}\right)}{\left\|u_{n}^{+}\right\|_{\infty}^{p}} \mathrm{~d} t \leq \frac{1}{p} \int_{\left\{y^{+} \neq 0\right\}} \vartheta\left(y^{+}\right)^{p} \mathrm{~d} t .
$$

We return to (14), take limits as $n \rightarrow \infty$ and use (11) and (19). We obtain

$$
\frac{1}{p}\left\|\left(y^{+}\right)^{\prime}\right\|_{p}^{p} \leq \frac{1}{p} \int_{\left\{y^{+} \neq 0\right\}} \vartheta\left(y^{+}\right)^{p} \mathrm{~d} t .
$$

If $y^{+}=0$, then from (12) in the limit as $n \rightarrow \infty$, we have

$$
\frac{1}{p}\left\|y^{-}\right\|^{p} \leq 0, \text { i.e., } y^{-}=0 \text {. }
$$

Therefore $y=0$, a contradiction to (11).

So, $y^{+} \neq 0$. Then from (20) and since in (4) the minimized function is $p$ homogeneous, it follows that $\widehat{\lambda}_{0}(\vartheta) \leq 0$, a contradiction to hypothesis $\mathrm{H}(\mathrm{iii})$. This proves that $\widehat{\varphi}$ is coercive.

Proposition 3.4. If hypotheses $H$ hold, then $\widehat{\varphi}$ is sequentially weakly lower semicontinuous.

Proof. Recall that in a Banach space the norm functional is sequentially weakly lower semicontinuous and by the Sobolev embedding theorem $W_{\text {per }}^{1, p}(0, b)$ is embedded compactly into $C(T)$. Therefore, in order to show the sequential weak lower semicontinuity of $\widehat{\varphi}$, it suffices to show that the integral functional $I_{\widehat{F}}: W_{\text {per }}^{1, p}(0, b) \longrightarrow \mathbb{R}$ defined by $I_{\widehat{F}}(u)=-\int_{0}^{b} \widehat{F}(t, u(t)) \mathrm{d} t$ for all $u \in W_{\text {per }}^{1, p}(0, b)$, is sequentially weakly 
lower semicontinuous. To this end, let $u_{n} \stackrel{w}{\longrightarrow} u$ in $W_{\text {per }}^{1, p}(0, b)$. Then $u_{n} \longrightarrow u$ in $C(T)$ and so $u_{n}^{ \pm} \longrightarrow u^{ \pm}$in $C(T)$. We have:

$$
\begin{aligned}
& -\int_{0}^{b} \widehat{F}\left(t, u_{n}\right) \mathrm{d} t \\
= & -\int_{0}^{b} F\left(t, u_{n}^{+}\right) \mathrm{d} t-\frac{1}{p}\left\|u_{n}^{+}\right\|_{p}^{p}-\int_{0}^{b} f(t, 0)\left(-u_{n}^{-}\right) \mathrm{d} t \text { for all } n \geq 1
\end{aligned}
$$

(see (5)).

Note that

$$
\frac{1}{p}\left\|u_{n}^{+}\right\|_{p}^{p} \longrightarrow \frac{1}{p}\left\|u^{+}\right\|_{p}^{p} \text { and } \int_{0}^{b} f(t, 0)\left(-u_{n}^{-}\right) \mathrm{d} t \longrightarrow \int_{0}^{b} f(t, 0)\left(-u^{-}\right) \mathrm{d} t .
$$

Also, (13) permits the use of Fatou's lemma and we have

$$
\begin{aligned}
& \liminf _{n \rightarrow \infty}\left(-\int_{0}^{b} F\left(t, u_{n}^{+}\right) \mathrm{d} t\right)=-\limsup _{n \rightarrow \infty} \int_{0}^{b} F\left(t, u_{n}^{+}\right) \mathrm{d} t \\
\geq & -\int_{0}^{b} \limsup _{n \rightarrow \infty} F\left(t, u_{n}^{+}\right) \mathrm{d} t \\
= & -\int_{0}^{b} F\left(t, u^{+}\right) \mathrm{d} t .
\end{aligned}
$$

From (21) through (23) it follows that

$$
\begin{aligned}
\liminf _{n \rightarrow \infty} I_{\widehat{F}}\left(u_{n}\right) & \geq-\int_{0}^{b} F\left(t, u^{+}\right) \mathrm{d} t-\frac{1}{p}\left\|u^{+}\right\|_{p}^{p}-\int_{0}^{b} f(t, 0)\left(-u^{-}\right) \mathrm{d} t=I_{\widehat{F}}(u), \\
& \Rightarrow I_{\widehat{F}}(\cdot) \text { is sequentially weakly lower semicontinuous, } \\
& \Rightarrow \widehat{\varphi} \text { is sequentially weakly lower semicontinuous. }
\end{aligned}
$$

Next we prove the differentiability of the functional $\widehat{\varphi}$.

Proposition 3.5. If hypotheses $H$ hold, then $\widehat{\varphi} \in C^{1}\left(W_{p e r}^{1, p}(0, b)\right)$.

Proof. From the definition of $\widehat{\varphi}$ it is clear that it suffices to show that the functional $u \longrightarrow \int_{0}^{b} \widehat{F}(t, u(t)) \mathrm{d} t, u \in W_{\text {per }}^{1, p}(0, b)$ is $C^{1}$. To this end let $u, h \in W_{\text {per }}^{1, p}(0, b)$ and let

$$
w(h)=\int_{0}^{b}(\widehat{F}(t, u+h)-\widehat{F}(t, u)-\widehat{f}(t, u) h) \mathrm{d} t .
$$

We have

$$
\begin{aligned}
\widehat{F}(t, u(t)+h(t))-\widehat{F}(t, u(t)) & =\int_{0}^{1} \frac{\mathrm{d}}{\mathrm{d} r} \widehat{F}(t, u(t)+r h(t)) \mathrm{d} r \\
& =\int_{0}^{1} \widehat{f}(t, u(t)+r h(t)) h(t) \mathrm{d} r
\end{aligned}
$$


Therefore

$$
|w(h)| \leq \int_{0}^{b} \int_{0}^{1}|\widehat{f}(t, u(t)+r h(t))-\widehat{f}(t, u(t))||h(t)| \mathrm{d} r \mathrm{~d} t .
$$

Because of (5), we have

$$
\widehat{f}(t, u(t)+r h(t))= \begin{cases}f(t, 0) & \text { if } t \in\{u+r h \leq 0\} \\ f(t, u(t)+r h(t))+(u(t)+r h(t))^{p-1} & \text { if } t \in\{u+r h>0\}\end{cases}
$$

By virtue of hypothesis $\mathrm{H}(\mathrm{ii})$ for a.a. $t \in\{u+r h>0\}$ and all $r \in[0,1]$, we have

$$
\begin{aligned}
f(t, u(t)+r h(t)) & \geq f\left(t,\|u+r h\|_{\infty}\right) \frac{(u(t)+r h(t))^{p-1}}{\|u+r h\|_{\infty}^{p-1}} \\
& \geq \frac{f\left(t,\|u\|_{\infty}+\|h\|_{\infty}\right)}{\|u\|_{\infty}+\|h\|_{\infty}}(u(t)+r h(t))^{p-1}(\text { recall } r \in[0,1]) \\
& \geq-2^{p-2} \frac{\left|f\left(t,\|u\|_{\infty}+\|h\|_{\infty}\right)\right|}{\|u\|_{\infty}+\|h\|_{\infty}}\left(\|u\|_{\infty}^{p-1}+\|h\|_{\infty}^{p-1}\right), \\
& \Rightarrow f(t, u(t)+r h(t)) \geq \alpha_{3}(t)
\end{aligned}
$$

for a.a. $t \in\{u+r h>0\}$, all $r \in[0,1]$, with $\alpha_{3} \in L^{1}(T)$.

In addition, hypothesis $\mathrm{H}(\mathrm{i})$ implies that for a.a. $t \in\{u+r h>0\}$, all $r \in[0,1]$, we have

$$
\begin{aligned}
f(t, u(t)+r h(t)) & \leq \alpha(t)\left(1+(u(t)+r h(t))^{p-1}\right) \\
& \leq \alpha(t)\left(1+2^{p-2}\left(\|u\|_{\infty}+\|h\|_{\infty}\right)\right) \\
& \Rightarrow f(t, u(t)+r h(t)) \leq \alpha_{4}(t)
\end{aligned}
$$

for a.a. $t \in\{u+r h>0\}$, all $r \in[0,1]$, with $\alpha_{4} \in L^{1}(T)$.

Recalling that $f(\cdot, 0) \in L^{1}(T)$, from (25) through (27), we infer that

(28) $|\widehat{f}(t, u(t)+r h(t))| \leq \alpha_{5}(t)$ for a.a. $t \in T$, all $r \in[0,1]$, with $\alpha_{5} \in L^{1}(T)$.

From (24) we have

$$
\begin{aligned}
|w(h)| \leq & \int_{0}^{b} \int_{0}^{1}|\widehat{f}(t, u(t)+r h(t))-\widehat{f}(t, u(t))|\|h\|_{\infty} \mathrm{d} r \mathrm{~d} t \\
\leq & c_{3} \int_{0}^{1} \int_{0}^{b}|\widehat{f}(t, u(t)+r h(t))-\widehat{f}(t, u(t))| \mathrm{d} t \mathrm{~d} r\|h\| \\
& \quad \text { for some } c_{3}>0 \quad \text { (by Fubini's theorem) } \\
\leq & c_{3} \int_{0}^{1}\left\|N_{\widehat{f}}(u+r h)-N_{\widehat{f}}(u)\right\|_{1} \mathrm{~d} r\|h\|
\end{aligned}
$$


where $N_{\widehat{f}}(v)(\cdot)=\widehat{f}(\cdot, v(\cdot))$ for all $v \in W_{\text {per }}^{1, p}(0, b)$.

From (28) and the dominated convergence theorem, we see that

$$
\begin{aligned}
& \int_{0}^{1}\left\|N_{\widehat{f}}(u+r h)-N_{\widehat{f}}(u)\right\|_{1} \mathrm{~d} r \longrightarrow 0 \text { as }\|h\| \rightarrow \infty, \\
\Rightarrow & \frac{|w(h)|}{\|h\|} \longrightarrow 0 \text { as }\|h\| \rightarrow \infty \\
\Rightarrow & \widehat{\varphi}^{\prime}(u)=A(u)-N_{\widehat{f}}(u) .
\end{aligned}
$$

But $A(\cdot)$ is continuous and from the above argument it is clear that $N_{\widehat{f}}(\cdot)$ is continuous too. Therefore $\widehat{\varphi} \in C^{1}\left(W_{\text {per }}^{1, p}(0, b)\right)$.

Now we are ready to produce nontrivial positive solutions for problem problem (1).

Proposition 3.6. If hypotheses $H$ hold, then problem (1) has a solution $u_{0} \in$ $\widehat{C}_{+} \backslash\{0\}$.

Proof. Propositions 3.3, 3.4, and the Weierstrass theorem imply that there is a $u_{0} \in W_{\text {per }}^{1, p}(0, b)$ such that

$$
\widehat{\varphi}\left(u_{0}\right)=\inf \left[\widehat{\varphi}(u): u \in W_{\text {per }}^{1, p}(0, b)\right]=\widehat{m} .
$$

Note that, if $u_{0}^{-} \neq 0$, then

$$
\begin{aligned}
\widehat{\varphi}\left(u_{0}^{+}\right)= & \frac{1}{p}\left\|\left(u_{0}^{+}\right)^{\prime}\right\|_{p}^{p}+\frac{1}{p}\left\|u_{0}^{+}\right\|_{p}^{p}-\int_{0}^{b} \widehat{F}\left(t, u_{0}^{+}\right) \mathrm{d} t \\
= & \frac{1}{p}\left\|\left(u_{0}^{+}\right)^{\prime}\right\|_{p}^{p}-\int_{0}^{b} F\left(t, u_{0}^{+}\right) \mathrm{d} t \quad(\text { see }(5)) \\
< & \frac{1}{p}\left\|u_{0}^{\prime}\right\|_{p}^{p}+\frac{1}{p}\left\|u_{0}^{-}\right\|_{p}^{p}-\int_{0}^{b} F\left(t, u_{0}^{+}\right) \mathrm{d} t-\int_{0}^{b} f(t, 0)\left(-u_{0}^{-}\right) \mathrm{d} t \\
& (\text { see (9)) } \\
= & \widehat{\varphi}\left(u_{0}\right),
\end{aligned}
$$

which contradicts (29) (recall $u_{0}^{+} \in W_{\text {per }}^{1, p}(0, b)$ ). Therefore $u_{0}^{-}=0$ and so $u_{0} \geq 0$.

Next we show that $u_{0} \neq 0$. By virtue of hypothesis H(iv) and the definition of $\widehat{\lambda}_{1}\left(\vartheta_{0}\right)$ (see (4)), we see that we can find $\widehat{u} \in W_{\text {per }}^{1, p}(0, b)$ such that

$$
\left\|\widehat{u}^{\prime}\right\|_{p}^{p}-\int_{\{\widehat{u} \neq 0\}} \vartheta_{0}|\widehat{u}|^{p} \mathrm{~d} t<0 \text { and }\|\widehat{u}\|_{p}=1
$$

Replacing $\widehat{u}$ with $|\widehat{u}| \in W_{\text {per }}^{1, p}(0, b)$ if necessary, we may assume that $\widehat{u} \geq 0, \widehat{u} \neq 0$ (see (30)). 
For $x>0$, we have

$$
\begin{aligned}
F(t, x) & =\int_{0}^{1} \frac{\mathrm{d}}{\mathrm{d} r} F(t, r x) \mathrm{d} r=\int_{0}^{1} f(t, r x) x \mathrm{~d} r \\
& \Rightarrow \frac{F(t, x)}{x^{p}}=\int_{0}^{1} \frac{f(t, r x)}{x^{p-1}} \mathrm{~d} r \geq \frac{f(t, x)}{x^{p-1}} \int_{0}^{1} r^{p-1} \mathrm{~d} r=\frac{1}{p} \frac{f(t, x)}{x^{p-1}}
\end{aligned}
$$

(see hypothesis $\mathrm{H}(\mathrm{ii})$ ),

$$
\Rightarrow \liminf _{x \rightarrow 0^{+}} \frac{F(t, x)}{x^{p}} \geq \frac{1}{p} \vartheta_{0}(t) \text { for a.a. } t \in T .
$$

For $r \in(0,1]$ small, we will have $r \widehat{u}(t) \in[0,1]$ for all $t \in T$. Then

$$
\frac{F(t, r \widehat{u}(t))}{r^{p}}=\frac{1}{r^{p}} \int_{0}^{r \widehat{u}(t)} f(t, s) \mathrm{d} s \geq \frac{1}{r^{p}} \int_{0}^{r \widehat{u}(t)} f(t, 1) s^{p-1} \mathrm{~d} s
$$

(see hypothesis $\mathrm{H}(\mathrm{ii})$ ),

(32)

$$
\begin{aligned}
& \geq \frac{1}{p} f(t, 1) \widehat{u}(t)^{p} \\
& \geq-\frac{1}{p} f(t, 1)\|\widehat{u}\|_{\infty}^{p} .
\end{aligned}
$$

By hypothesis $-\frac{1}{p} f(\cdot, 1)\|\widehat{u}\|_{\infty}^{p} \in L^{1}(T)$. Because of (32), we can apply Fatou's lemma and using (31), we obtain

$$
\begin{aligned}
& \liminf _{r \rightarrow 0^{+}} \int_{\{\widehat{u} \neq 0\}} \frac{F(t, r \widehat{u})}{r^{p}} \mathrm{~d} t \geq \frac{1}{p} \int_{\{\widehat{u} \neq 0\}} \vartheta_{0} \widehat{u}^{p} \mathrm{~d} t \\
\Rightarrow & \frac{1}{p}\left\|\widehat{u}^{\prime}\right\|_{p}^{p}-\int_{0}^{b} \frac{F(t, r \widehat{u})}{r^{p}} \mathrm{~d} t<0 \text { for } r \in(0,1) \text { small } \quad(\text { see }(30)), \\
\Rightarrow & \widehat{\varphi}(r \widehat{u})<0 \text { for } r \in(0,1) \text { small } \quad(\text { recall } \widehat{u} \geq 0), \\
\Rightarrow & \widehat{m}=\widehat{\varphi}\left(u_{0}\right)<0=\widehat{\varphi}(0) \quad(\text { see }(29)), \\
\Rightarrow & u_{0} \neq 0 .
\end{aligned}
$$

From (29) and Proposition 3.5, we have

$$
\begin{aligned}
& \widehat{\varphi}^{\prime}\left(u_{0}\right)=0, \\
\Rightarrow & A\left(u_{0}\right)=N_{f}\left(u_{0}\right) \text { with } N_{f}(u)(\cdot)=f(\cdot, u(\cdot)) \text { for all } u \in W_{\text {per }}^{1, p}(0, b) \\
& \left(\text { recall } u_{0} \geq 0 \text { and see }(5)\right), \\
\Rightarrow & \left\{\begin{array}{c}
-\left(\left|u_{0}^{\prime}(t)\right|^{p-2} u_{0}^{\prime}(t)\right)^{\prime}=f\left(t, u_{0}(t)\right) \text { a.e. on } T, \\
u_{0}(0)=u_{0}(b), u_{0}^{\prime}(0)=u_{0}^{\prime}(b) \text { with } u_{0} \in C^{1}(T)
\end{array}\right\} \\
& (\text { see Kyritsi-Papageorgiou [7]). }
\end{aligned}
$$


In fact we can improve the conclusion of this proposition, by strengthening a little hypothesis $\mathrm{H}(\mathrm{i})$. So, the new hypotheses on $f(t, x)$ are:

$\underline{\mathbf{H}^{\prime}}: f: T \times \mathbb{R} \longrightarrow \mathbb{R}$ is a Caratheodory function such that

(i) for all $x \geq 0$, there exists $\widehat{M}>0$ such that for all $x \geq \widehat{M}, f(\cdot, x) \in L^{\infty}(T)$ and

$$
f(t, x) \leq \alpha(t)\left(1+x^{p-1}\right) \text { for a.a } t \in T, \text { all } x \geq 0, \text { with } \alpha \in L^{1}(T)_{+} ;
$$

hypotheses $\mathrm{H}^{\prime}$ (ii), (iii), (iv) are the same as the corresponding hypotheses $\mathrm{H}(\mathrm{ii})$, (iii), (iv).

Proposition 3.7. If hypotheses $H^{\prime}$ hold, then problem (1) has a solution $u_{0} \in$ int $\widehat{C}_{+}$.

Proof. From Proposition 3.6 we already have a positive solution $u_{0} \in \widehat{C}_{+} \backslash\{0\}$.

By virtue of hypothesis $\mathrm{H}^{\prime}(\mathrm{ii})$, for $\xi \geq \max \left\{\widehat{M},\left\|u_{0}\right\|_{\infty}\right\}$ and for a.a. $t \in\left\{u_{0}>0\right\}$ we have

$$
f\left(t, u_{0}(t)\right) \geq \frac{f(t, \xi)}{\xi^{p-1}} u_{0}(t)^{p-1} \geq-c_{3} u_{0}(t)^{p-1} \text { for some } c_{3}>0
$$

(see hypothesis $\mathrm{H}^{\prime}(\mathrm{ii})$ ).

Therefore from (33) we have

$$
\begin{aligned}
& \left(\left|u_{0}^{\prime}(t)\right|^{p-2} u_{0}^{\prime}(t)\right)^{\prime} \leq c_{3} u_{0}^{\prime}(t)^{p-1} \text { a.e. on } T \\
& \left(\text { recall } u_{0}^{\prime}(t)=0 \text { a.e. on } T\left\{u_{0}=0\right\},\right. \text { see [9]), } \\
\Rightarrow & u_{0} \in \operatorname{int} \widehat{C}_{+} \quad \text { (by Vazquez [10]). }
\end{aligned}
$$

\section{Uniqueness of Positive Solutions}

In this section we establish the uniqueness of the positive solution. In fact we show that hypotheses $\mathrm{H}^{\prime}$ (iii) and (iv) are both necessary and sufficient for the existence and uniqueness of a positive solution for problem (1).

Proposition 4.1. If hypotheses $H^{\prime}$ hold, then problem (1) has a unique positive solution $u_{0} \in \operatorname{int} \widehat{C}_{+}$.

Proof. Let $u, v \in \widehat{C}_{+} \backslash\{0\}$ be two positive solutions for problem (1). From the proof of Proposition 3.7, we have that $u, v \in \operatorname{int} \widehat{C}_{+}$. So, 


$$
\begin{aligned}
& \int_{0}^{b} \frac{f(t, u)}{u^{p-1}}\left(u^{p}-v^{p}\right) \mathrm{d} t \\
= & -\int_{0}^{b}\left(\left|u^{\prime}\right|^{p-2} u^{\prime}\right)^{\prime}\left(u-\frac{v^{p}}{u^{p-1}}\right) \mathrm{d} t \\
= & \int_{0}^{b}\left|u^{\prime}\right|^{p-2} u^{\prime}\left(u^{\prime}-\left(\frac{v^{p}}{u^{p-1}}\right)^{\prime}\right) \mathrm{d} t \quad \text { (by integration by parts) } \\
= & \left\|u^{\prime}\right\|_{p}^{p}-\int_{0}^{b}\left|u^{\prime}\right|^{p-2} u^{\prime}\left(\frac{v^{p}}{u^{p-1}}\right)^{\prime} \mathrm{d} t \\
= & \left\|u^{\prime}\right\|_{p}^{p}-\left\|v^{\prime}\right\|_{p}^{p}+\int_{0}^{b} R(v, u) \mathrm{d} t \quad \text { (see Section 2). }
\end{aligned}
$$

Similarly interchanging the roles of $u$ and $v$, we obtain

$$
\int_{0}^{b} \frac{f(t, v)}{v^{p}}\left(v^{p}-u^{p}\right) \mathrm{d} t=\left\|v^{\prime}\right\|_{p}^{p}-\left\|u^{\prime}\right\|_{p}^{p}+\int_{0}^{b} R(u, v) \mathrm{d} t .
$$

Adding (34) and (35), we have

$$
0 \geq \int_{0}^{b}\left(\frac{f(t, u)}{u^{p-1}}-\frac{f(t, v)}{v^{p-1}}\right)\left(u^{p}-v^{p}\right) \mathrm{d} t=\int_{0}^{b}[R(v, u)+R(u, v)] \mathrm{d} t \geq 0
$$

(see hypothesis $\mathrm{H}^{\prime}$ (ii) and recall $R(u, v), R(v, u) \geq 0$ ).

It follows that $R(u, v)=R(v, u)=0$ and so $u=k v$ for some $k>0$ (see Allegretto-Huang [2]). The fact that for a.a. $t \in T, x \longrightarrow \frac{f(t, x)}{x^{p-1}}$ is strictly decreasing, (see hypothesis $\mathrm{H}^{\prime}(\mathrm{ii})$ ), implies that $k=1$ and so $u=v$. This proves the uniqueness of the positive solution $u_{0} \in \operatorname{int} \widehat{C}_{+}$.

As we already mentioned, hypotheses $\mathrm{H}^{\prime}$ (iii) and (iv) are also necessary for the uniqueness of the positive solution $u_{0} \in \operatorname{int} \widehat{C}_{+}$.

Proposition 4.2. If $f: T \times \mathbb{R} \longrightarrow \mathbb{R}$ is a Caratheodory function satisfying hypotheses $H^{\prime}(i)$, (ii), and problem (1) has a unique positive solution $u_{0} \in \widehat{C}_{+} \backslash\{0\}$, then $\widehat{\lambda}_{0}\left(\vartheta_{0}\right)<0<\widehat{\lambda}_{0}(\vartheta)$ where

$$
\vartheta_{0}(t)=\lim _{x \rightarrow 0^{+}} \frac{f(t, x)}{x^{p-1}} \text { and } \vartheta(t)=\lim _{x \rightarrow+\infty} \frac{f(t, x)}{x^{p-1}} .
$$

Proof. From Proposition 3.7, we know that $u_{0} \in \operatorname{int} \widehat{C}_{+}$. We have

$$
\widehat{\lambda}_{0}\left(\vartheta_{0}\right) \leq \frac{\left\|u_{0}^{\prime}\right\|_{p}^{p}-\int_{0}^{b} \vartheta_{0} u_{0}^{p} \mathrm{~d} t}{\left\|u_{0}\right\|_{p}^{p}} \quad\left(\text { see (4) and recall } u_{0}(t)>0 \text { for all } t \in T\right)
$$




$$
\begin{aligned}
& =\frac{\int_{0}^{b} f\left(t, u_{0}\right) u_{0} \mathrm{~d} t-\int_{0}^{b} \vartheta_{0} u_{0}^{p} \mathrm{~d} t}{\left\|u_{0}\right\|_{p}^{p}} \\
& \left.<\frac{\int_{0}^{b} \vartheta_{0} u_{0}^{p} \mathrm{~d} t-\int_{0}^{b} \vartheta_{0} u_{0}^{p} \mathrm{~d} t}{\left\|u_{0}\right\|_{p}^{p}}=0 \quad \text { (see hypothesis } \mathrm{H}^{\prime}(\mathrm{ii})\right) .
\end{aligned}
$$

So, we have proved that $\widehat{\lambda}_{0}\left(\vartheta_{0}\right)<0$.

Let $\beta(t)=\frac{f\left(t,\left\|u_{0}\right\|_{\infty}+1\right)}{\left(\left\|u_{0}\right\|_{\infty}+1\right)^{p-1}}$. Then $\beta \in L^{1}(T)$ (see hypothesis $\mathrm{H}^{\prime}(\mathrm{i})$ ). Let $\widehat{u}_{1} \in$ int $\widehat{C}_{+}$be the $L^{p}$-normalized eigenfunction corresponding to the eigenvalue $\widehat{\lambda}_{0}(\beta)$ (see Binding-Rynne [4]). For $k>0$ large enough we will have $u_{0}<k \widehat{u}_{1}=\widetilde{u}_{1}$. As in the proof of Proposition 4.1, we show that

$$
\begin{gathered}
\int_{0}^{b} \frac{f\left(t, u_{0}\right)}{u_{0}^{p-1}}\left(u_{0}^{p}-\widetilde{u}_{1}^{p}\right) \mathrm{d} t=\left\|u_{0}^{\prime}\right\|_{p}^{p}-\left\|\widetilde{u}_{1}^{\prime}\right\|_{p}^{p}+\int_{0}^{b} R\left(\widetilde{u}_{1}, u_{0}\right) \mathrm{d} t . \\
\int_{0}^{b}\left(\widehat{\lambda}_{0}(\beta)+\beta\right)\left(\widetilde{u}_{1}^{p}-u_{0}^{p}\right) \mathrm{d} t=\left\|\widetilde{u}_{1}^{\prime}\right\|_{p}^{p}-\left\|u_{0}^{\prime}\right\|_{p}^{p}+\int_{0}^{b} R\left(u_{0}, \widetilde{u}_{1}\right) \mathrm{d} t .
\end{gathered}
$$

We add (36) and (37). Then

(38) $\int_{0}^{b}\left(\frac{f\left(t, u_{0}\right)}{u_{0}^{p-1}}-\left(\widehat{\lambda}_{0}(\beta)+\beta\right)\right)\left(u_{0}^{p}-\widetilde{u}_{1}^{p}\right) \mathrm{d} t=\int_{0}^{b}\left[R\left(\widetilde{u}_{1}, u_{0}\right)+R\left(u_{0}, \widetilde{u}_{1}\right)\right] \mathrm{d} t \geq 0$.

By virtue of hypothesis $\mathrm{H}^{\prime}$ (ii) we have

$$
\begin{aligned}
\frac{f\left(t, u_{0}\right)}{u_{0}^{p-1}} & >\frac{f\left(t,\left\|u_{0}\right\|_{\infty}+1\right)}{\left(\left\|u_{0}\right\|_{\infty}+1\right)^{p-1}}=\beta(t) \text { a.e. on } T, \\
& \Rightarrow \frac{f\left(t, u_{0}\right)}{u_{0}^{p-1}}-\beta(t)>0 \text { a.e. on } T \text {. }
\end{aligned}
$$

Also since $u_{0}<\widetilde{u}_{1}$, we have

$$
\left(u_{0}^{p}-\widetilde{u}_{1}^{p}\right)(t)<0 \text { for all } t \in T .
$$

Using (39) and (40) in (38), we infer that $\widehat{\lambda}_{0}(\beta)>0$. But note that $\beta \geq \vartheta$ (see hypothesis $\left.\mathrm{H}^{\prime}(\mathrm{ii})\right)$ and so from (4) we have that $0<\widehat{\lambda}_{0}(\beta) \leq \widehat{\lambda}_{0}(\vartheta)$.

Summarizing the situation, we have the following definitive existence and uniqueness theorem for problem (1). 
Theorem 4.3. If $f: T \times \mathbb{R} \longrightarrow \mathbb{R}$ is a Caratheodory function satisfying hypotheses $H^{\prime}(i)$, (ii), then problem (1) has a unique positive solution $u_{0} \in$ int $\widehat{C}_{+}$

$$
\text { if and only if }
$$

$$
\widehat{\lambda}_{0}\left(\vartheta_{0}\right)<0<\widehat{\lambda}_{0}(\vartheta)
$$

where $\vartheta_{0}(t)=\lim _{x \rightarrow 0^{+}} \frac{f(t, x)}{x^{p-1}}$ and $\vartheta(t)=\lim _{x \rightarrow+\infty} \frac{f(t, x)}{x^{p-1}}$.

\section{ACKNOWLEDGMENTS}

The authors wish to thank a very knowledgeable for his/her corrections and remarks.

\section{REFERENCES}

1. S. Aizicovici, N. S. Papageorgiou and V. Staicu, Multiple nontrival solutions for nonlinear periodic problems with the $p$-Laplacian, J. Diff. Eqns., 243 (2007), 504-535.

2. W. Allegretto and Y. Huang, A Picone's identity for the $p$-Laplacian and applications, Nonlinear Analysis, 32 (1998), 819-830.

3. P. Binding and B. P. Rynne, The spectrum of the periodic $p$-Laplacian, J. Differential Equations, 235 (2007), 199-218.

4. P. Binding and B. P. Rynne, Variational and nonvariational eigenvalues of the $p$-Laplacian, J. Differential Equations, 244 (2008), 24-39.

5. M. del Pino, M. A.-R. Manásevich and A. Murúa, Existence and multiplicity of solutions with prescribed period for a second order quasilinear ODE, Nonlinear Analysis, 18 (1992), 79-92.

6. P. Drabek and R. Manásevich, On the closed solution to some nonhomogeneous eigenvalue problems with $p$-Laplacian, Differential Integral Eqns., 12 (1999), 773-788.

7. S. Th. Kyritsi and N. S. Papageorgiou, On the multiplicity of solutions for nonlinear periodic problems with the nonlinearity crossing several eigenvalues, Glasgow Math. J., 52 (2010), 271-302.

8. D. Motreanu, V. Motreanu and N. S. Papageorgiou, Multiple solutions for resonant periodic equations, Nonlinear Diff. Equ. Appl., 17 (2010), 535-557.

9. N. S. Papageorgiou and S. Th. Kyritsi-Yiallourou, Handbook of Applied Analysis, Springer, New York, 2009.

10. J. Vazquez, A strong maximum principle for some quasilinear elliptic equations, Appl. Math. Optim., 12 (1984), 191-202.

11. X. Yang, Multiple periodic solutions of a class of $p$-Laplacian, J. Math. Anal. Appl., 314 (2006), 17-29.

12. M. Zhang, The roatation number approach to eigenvalues of the one-dimensional $p$ Laplacian with periodic potentials, J. London Math. Soc., 64 (2001), 125-143. 
Sophia Th. Kyritsi

Department of Mathematics

Hellenic Naval Academy

Piraeus 18539

Greece

E-mail: skyrits@math.ntua.gr

Nikolaos S. Papageorgiou

National Technical University

Department of Mathematics

Zografou Campus

Athens 15780

Greece

E-mail: npapg@math.ntua.gr 